Research Article

\title{
Prediction of Later-Age Concrete Compressive Strength Using Feedforward Neural Network
}

\author{
Thuy-Anh Nguyen, Hai-Bang Ly, Hai-Van Thi Mai $(\mathbb{D}$, and Van Quan Tran
}

University of Transport Technology, Hanoi 100000, Vietnam

Correspondence should be addressed to Hai-Van Thi Mai; vanmth@utt.edu.vn

Received 26 June 2020; Revised 11 August 2020; Accepted 18 August 2020; Published 8 September 2020

Guest Editor: Hainian Wang

Copyright (c) 2020 Thuy-Anh Nguyen et al. This is an open access article distributed under the Creative Commons Attribution License, which permits unrestricted use, distribution, and reproduction in any medium, provided the original work is properly cited.

\begin{abstract}
Accurate prediction of the concrete compressive strength is an important task that helps to avoid costly and time-consuming experiments. Notably, the determination of the later-age concrete compressive strength is more difficult due to the time required to perform experiments. Therefore, predicting the compressive strength of later-age concrete is crucial in specific applications. In this investigation, an approach using a feedforward neural network (FNN) machine learning algorithm was proposed to predict the compressive strength of later-age concrete. The proposed model was fully evaluated in terms of performance and prediction capability over statistical results of 1000 simulations under a random sampling effect. The results showed that the proposed algorithm was an excellent predictor and might be useful for engineers to avoid time-consuming experiments with the statistical performance indicators, namely, the Pearson correlation coefficient (R), root-mean-squared error (RMSE), and mean squared error (MAE) for the training and testing parts of $0.9861,2.1501,1.5650$ and $0.9792,2.8510,2.1361$, respectively. The results also indicated that the FNN model was superior to classical machine learning algorithms such as random forest and Gaussian process regression, as well as empirical formulations proposed in the literature.
\end{abstract}

\section{Introduction}

In the field of civil engineering, concrete plays a significant role and is the most important construction material [1-7]. Concrete is a kind of artificial stone material made by molding and solidifying an appropriate mixture of cement, water, coarse, and fine aggregate. To further enhance the properties of concrete, several chemical or mineral admixtures such as fly ash, blast furnace slag, silica fume, metakaolin, and superplasticizer could be added into the mixture. Compressive strength is the most important factor that reflects the quality of a concrete mix design [8]. The concrete compressive strength is usually characterized by estimating the values at 28 days. The compressive strength directly depends on the ingredients of concrete and their relative amount [9], making the procedure of selecting appropriate mixture components critical. Therefore, knowing the potential compressive strength of later-age concrete at the earliest possible time after curing, especially in the first hours, can be helpful for necessary adjustment.

In recent decades, the development of suitable tools to obtain the desired compressive strength has been the subject of numerous researches. Abrams' law is the well-known concept in the field of civil engineering for calculating the compressive strength of concrete with two inputs, namely, cement and water. The results of Abrams' law showed that an increase of compressive strength is inversely proportional to the water-to-cement ratio. Besides, the compressive strength of concrete also depends on the content and nature of the mix design, the curing condition, and the age of specimens [10-12]. Apart from Abrams' law, Hasan and Kabir [8] and ACI 209-71 [13] have proposed empirical equations to determine the compressive strength of concrete at any age (Table 1). However, the empirical methods could not take into account all factors that affect the concrete compressive strength, such as supplementary cementitious materials or 
TABLE 1: Summary of the empirical equations for determining the compressive strength of concrete.

\begin{tabular}{lccc}
\hline Ref. & Equation & Symbol & Time-dependent \\
\hline Abrams' law & $S=\left(A / B^{W / C}\right)$ & $S:$ compressive strength; $A, B:$ constant; $W / C$ : water/cement ratio & No \\
Hasan and Kabir [8] & $S_{t_{n}}=\left(p D_{n} /\left(D_{n}+q\right)\right)$ & $p, q$ : constant; $n$ : day; $D_{n}:$ number of days & Yes \\
ACI 209-71 [13] & $S_{t_{n}}=(t /(a+b t)) S_{28 \mathrm{~d}}$ & $a, b$ : constant; $t$ : number of days; $S_{28 \mathrm{~d}}:$ compressive strength at 28 days & Yes \\
\hline
\end{tabular}

the mixture components. Therefore, these methods cannot correctly estimate the compressive strength of concrete using supplementary cementitious materials. Alternative approach, thus, needs to be investigated to predict the laterage compressive strength of concrete accurately.

Recently, development and application of machine learning techniques in the civil engineering sector have gained extensive attention [14]. Taking advantage of an extensive experimental database, machine learning algorithms exhibit the ability to simplify classical approaches, such as experimental or numerical simulation. During the last four decades, machine learning techniques have been proven to successfully solve many problems of civil engineering such as coastal engineering, construction engineering [15], earthquake engineering, geotechnical engineering, environmental engineering, structural engineering [16, 17], and materials science engineering [18]. Moreover, taking into account numerous input factors is an essential advantage of machine learning techniques. Therefore, machine learning technique appears to be a potential approach to predict the later-age compressive strength of concrete. Among the machine learning algorithms that are currently being utilized, the feedforward neural network (FNN) is the most popular model due to the structural flexibility, excellent prediction performance, and the availability of a significant amount of training algorithms [19]. With the ability to deal with complex functions, FNN is an excellent predictor in many studies, such as compressive strength of concrete [20] or bearing capacity of steel tube columns [21].

In this paper, the FNN algorithm was developed and used to predict the later-age compressive strength of concrete. For this aim, a database containing 190 samples, gathered from the available literature, was used to train and validate the FNN algorithm. The quality of the FNN model was assessed by common statistical measurements such as root-mean-square error (RMSE), mean absolute error (MAE), and the Pearson correlation coefficient $(R)$. The paper is structured as follows: the introduction section is presented in Section 1. In Section 2, the machine learning feedforward neural network $(\mathrm{FNN})$ is introduced and fundamental elements are focused. Section 3 presents the database used for the development of FNN, as well as the statistical information of input and output variables. Section 4 shows the results obtained by FNN and emphasizes the superior prediction accuracy by comparing with classical machine learning models such as random forest and Gaussian process regression. Finally, Section 5 provides some conclusions and perspectives.

\section{Machine Learning Method}

2.1. Feedforward Neural Network. Feedforward neural network (FNN) is the most commonly used neural network in the literature [22]. It could be considered as one of the first and most successful machine learning algorithms. FNN could also be called a multilayer perceptron (MLP) or in a simple manner as neural networks. The FNN structure contains four main compositions: the input layer, output layer, one or more hidden layers, and the weights associated with each neuron in the hidden layer(s) [23]. In the input layer, it contains a certain number of neurons, which corresponds to the number of features in the dataset. The input layer receives the information related to the inputs and passes such information to the second layer, the hidden layer(s). The hidden layer is located between the input and output layers, which contains many neurons to apply the transformation from the input layer to the output layer. Each neuron in the hidden layer(s) is associated with a weight, representing the amplitude of a connection between two neurons. The output layer is the target of the problem or the feature that we want to predict [24].

The principle of the process of FNN can be summarized in 3 steps as follows: (i) multiplication of weights and inputs, where each feature in the input layer is multiplied by the weight associated; (ii) biases added: the product in the previous step is added, each with a bias; and (iii) activation step: an activation function is applied to map the features in the input layer to the output layer (Figure 1). Finally, the desired output is obtained by summing up all the results of the previous steps (for each neuron). The difference, or the error, of the predicted and actual outputs, is calculated and called a loss function, which measures the algorithm performance.

The effectiveness of FNN has been proven in many works $[25,26]$, especially in material modeling $[20,27]$. Therefore, FNN with ten neurons is proposed to predict the later-age compressive strength of concrete in this study. Detail information on the FNN, as well as all equations governing the algorithm, could be found in the literature [28].

2.2. Performance Criteria. In this study, the performance of FNN is evaluated by common statistical measurements such as the Pearson correlation coefficient $(R)$, root-mean-square error (RMSE), and mean absolute error (MAE). Typically, the values of $R$ are in the $[-1,1]$ range, and better prediction accuracy is obtained when the absolute value of $R$ is close to 1. Oppositely, lower values of RMSE and MAE designate a 


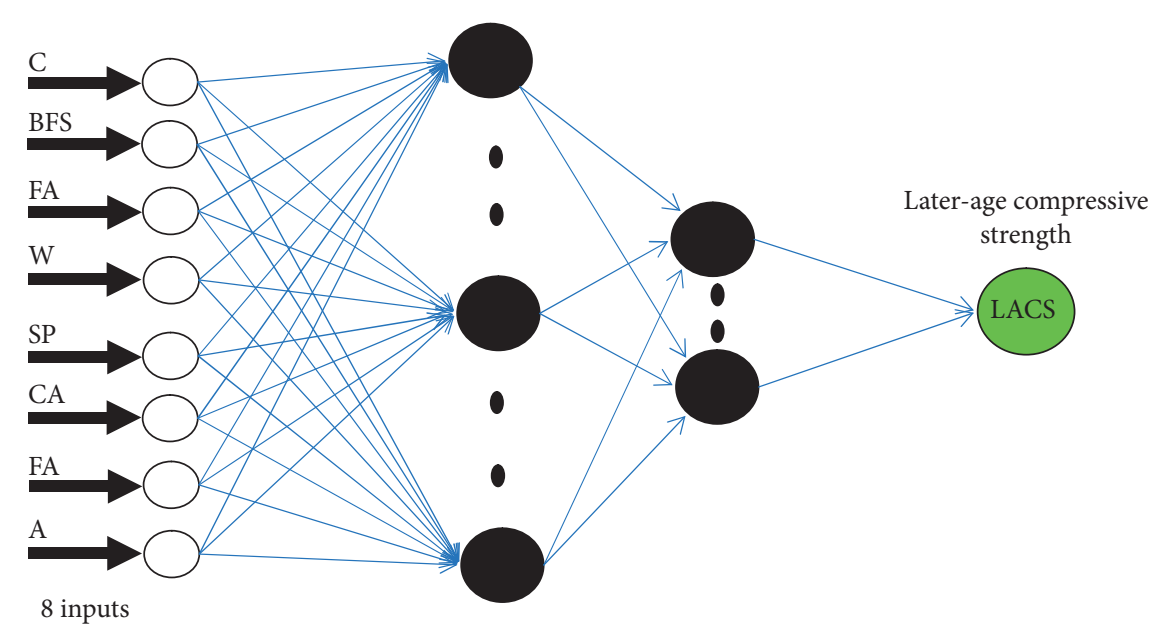

FIgURE 1: Illustration of the FNN algorithm used in this study, including 8 inputs.

higher performance of the given machine learning algorithm. The expressions of the indicators are as follows $[29,30]$ :

$$
\begin{aligned}
\text { MAE } & =\frac{1}{n} \sum_{i=1}^{n}\left(u_{i}-\bar{u}_{i}\right), \\
\mathrm{RMSE} & =\sqrt{\frac{1}{n} \sum_{i=1}^{n}\left(u_{i}-\bar{u}_{i}\right)^{2}} \\
R^{2} & =1-\frac{\sum_{i=1}^{n}\left(u_{i}-\bar{u}_{i}\right)^{2}}{\sum_{i=1}^{n}\left(u_{i}-\bar{u}\right)^{2}}
\end{aligned}
$$

where $\bar{u}_{i}$ is the predicted output value, $u_{i}$ is the measured target value, and $n$ is the number of samples.

\section{Database Collection}

In this study, a database containing 190 samples was collected from the literature [31-35]. The input parameters of the concrete compressive strength database include the content of cement (denoted as C), the content of blast furnace slag (denoted as BFS), the content of fly ash (denoted as FA), water content (denoted as W), superplasticizer (denoted as SP), aggregate content including coarse aggregates (denoted as CA) and fine aggregates (denoted as FA), and testing age (denoted as A). The considered output is the later-age compressive strength (LACS) of concrete.

For the statistical information of the database, Table 2 presents the min, average, max, median, and standard deviation values (denoted as $\mathrm{SD}$ ) of all the parameters used in this study.

The database containing 190 samples is divided randomly into two parts, namely, the training part (70\% of the total samples) and the testing part (30\% of the remaining samples). The training part is used to construct the FNN model, whereas the testing subset was dedicated to the assessment of the accuracy of the model.
TABle 2: Summary of the statistical information of the database used in the present work.

\begin{tabular}{lccccc}
\hline & Min & Average & Median & Max & SD \\
\hline C & 102 & 294.95 & 288.5 & 540 & 103 \\
BFS & 0 & 66.9 & 0 & 305.3 & 82.2 \\
FA & 0 & 31.93 & 0 & 174.7 & 55.8 \\
W & 121.8 & 190.19 & 192 & 228 & 25.8 \\
SP & 0 & 3.93 & 0 & 32.2 & 6 \\
CA & 852.1 & 979.73 & 968 & 1134 & 64.6 \\
FA & 594 & 762.22 & 780.35 & 993 & 98.5 \\
A & 90 & 146.75 & 100 & 365 & 90 \\
LACS & 21.86 & 49.97 & 44.21 & 82.6 & 12.8 \\
\hline
\end{tabular}

$\mathrm{SD}=$ standard deviation

\section{Results and Discussion}

4.1. Performance of FNN. The samples of the training part greatly affect the accuracy of the machine learning black box [36] so that the sampling technique is important in the construction phase of the model. A certain number of samples are randomly taken to be in the training part. Besides, there is systematic sampling, stratified sampling, or cluster sampling, belonging to the probability sampling technique. In this study, simple random sampling was chosen as it was an effective way to select samples. The performance of FNN is evaluated throughout the sampling technique in this section.

A number of 1000 simulations were performed in shuffling the indexes of the database, and $70 \%$ of data were taken to construct the training part. The results of 1000 simulations are given by the histograms of values $R$ (Figure 2), RMSE (Figure 3), and MAE (Figure 4) for the training and testing parts. The values of $R$ of the FNN algorithm showed a strong prediction capability of LACS as the minimum values of $R$ were in the $R=0.7-1$ range. Thus, a strong correlation was observed between the predicted output given by FNN and the experimental results.

The training dataset exhibited greater accuracy than the testing dataset, which was helpful in preventing overfitting problems. A high concentration of $R$ close to 1 was observed, 


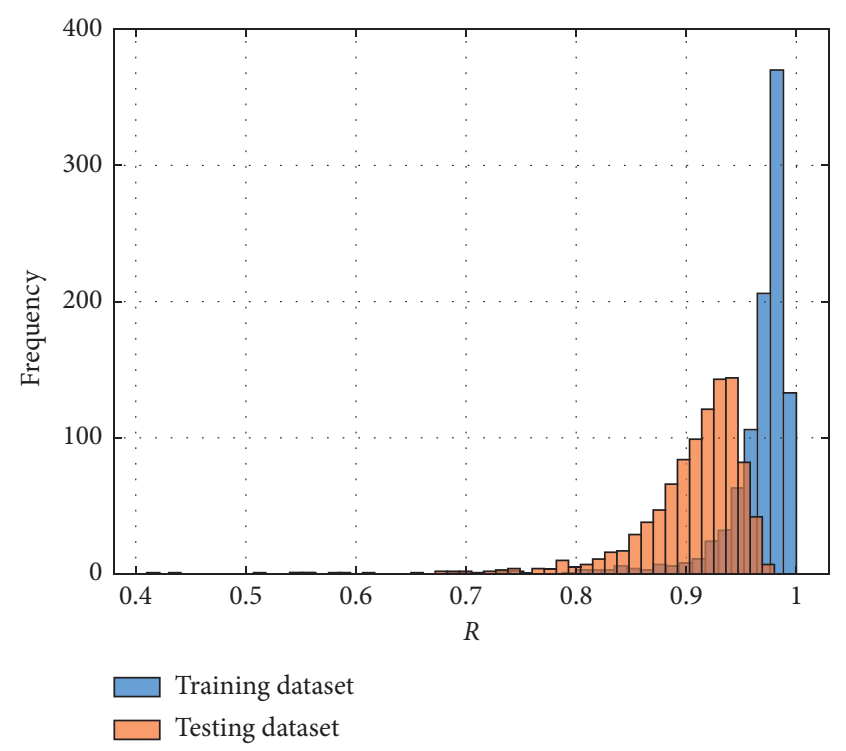

Figure 2: Histogram of $R$ values over 1000 simulations for the training and testing parts.

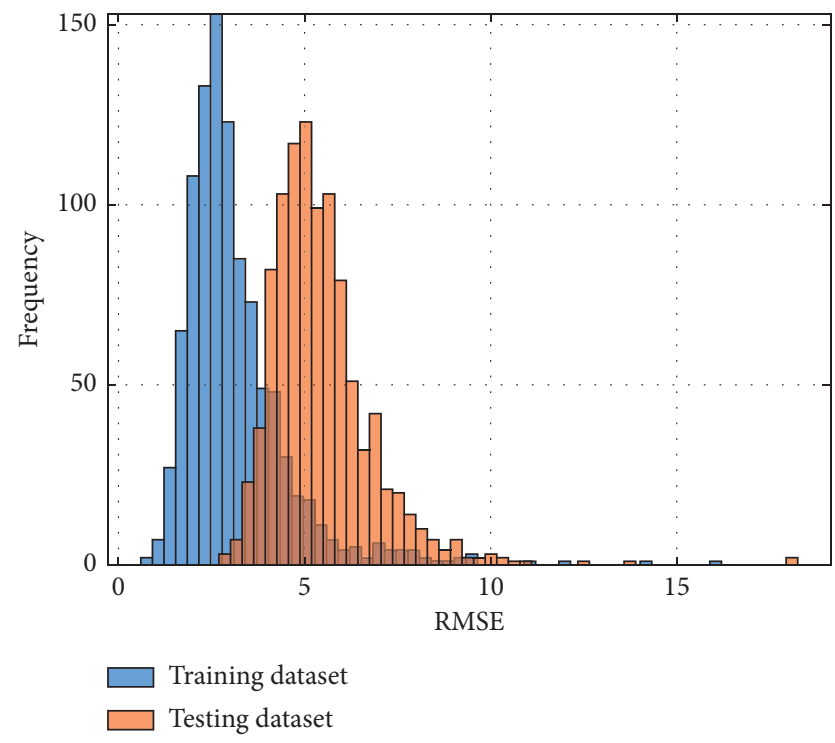

FIGURE 3: Histogram of RMSE values over 1000 simulations for the training and testing parts.

and $R=0.94$ was the most frequent result obtained for the testing dataset.

Similarly, the RMSE and MAE values showed complementary information on the observations. The most frequent values of RMSE and MAE were 2.5 and 2.1 for the training dataset, whereas those of the testing dataset were 5 and 3.9, respectively. In all the cases, no extreme values were observed for the training dataset, showing that the prediction performance of FNN was satisfactory. Table 3 shows the values of $R$, RMSE, and MAE for 1000 simulations performed in this study, along with the standard deviation values associated with each case. The average values of $R$ were 0.9391 and 0.9350 for the training and testing datasets,

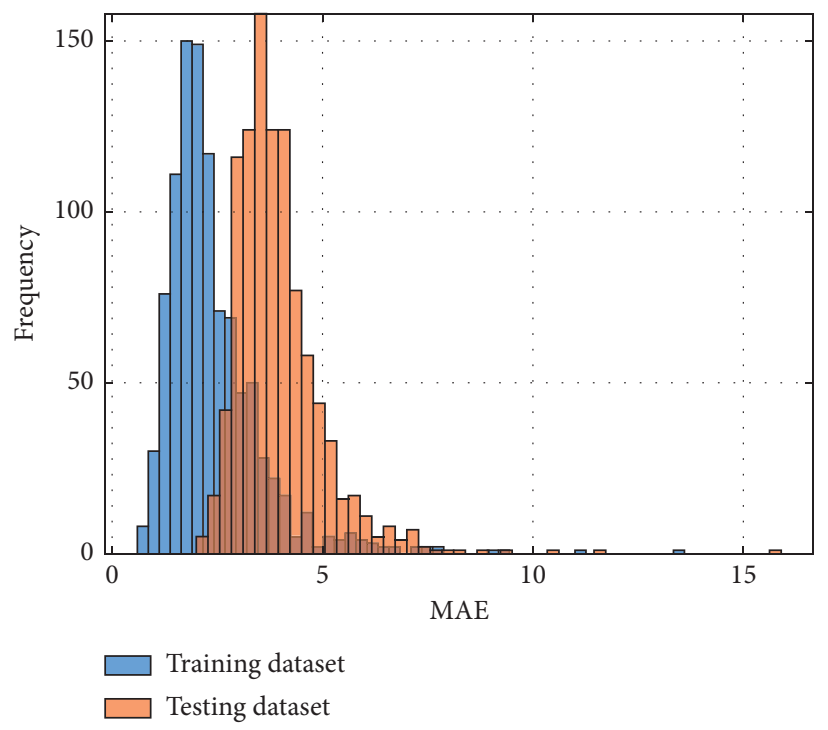

FIgURE 4: Histogram of MAE values over 1000 simulations for the training and testing parts.

TABLE 3: Statistical results over 1000 simulations using FNN in this study.

\begin{tabular}{lcccc}
\hline & Min & Average & Max & SD \\
\hline Training & & & & \\
$R$ & 0.8179 & 0.9391 & 0.9677 & 0.0233 \\
RMSE & 3.1481 & 4.1988 & 7.1676 & 0.7116 \\
MAE & 2.4860 & 3.0472 & 3.8312 & 0.3110 \\
\hline Testing & & & & \\
$R$ & 0.4379 & 0.9350 & 0.9781 & 0.0698 \\
RMSE & 2.7580 & 4.3342 & 11.5334 & 1.5287 \\
MAE & 2.2113 & 3.2552 & 7.5907 & 1.0247 \\
\hline
\end{tabular}

$\mathrm{SD}=$ standard deviation

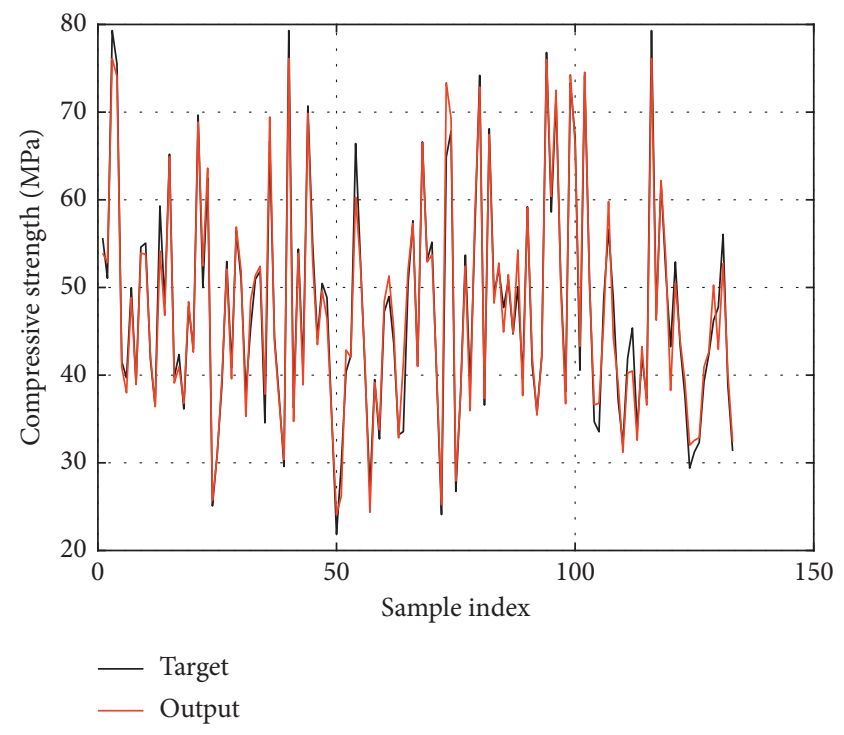

Figure 5: Prediction results of FNN for the training dataset. 


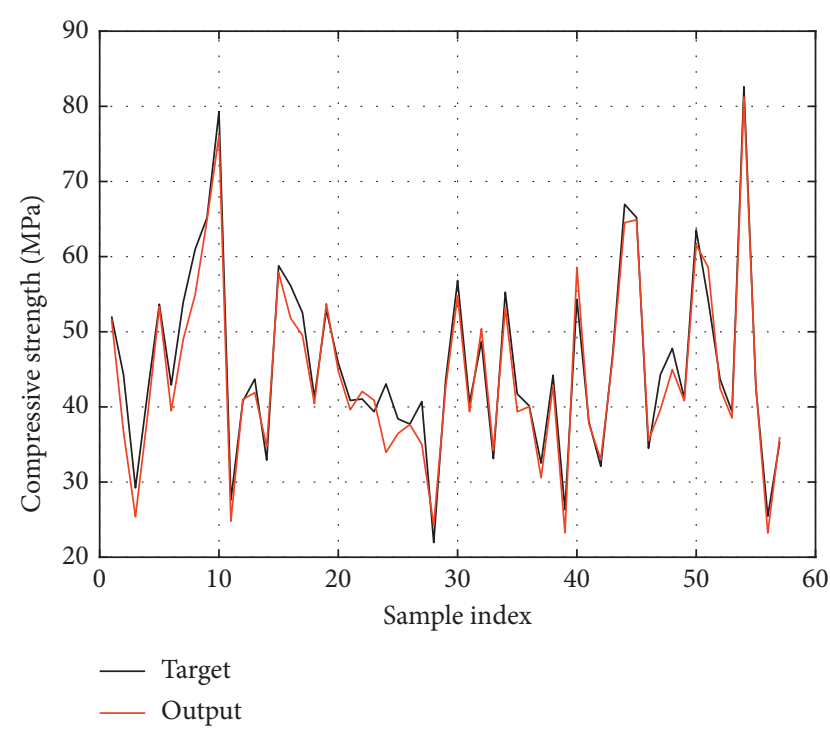

Figure 6: Prediction results of FNN for the testing dataset.

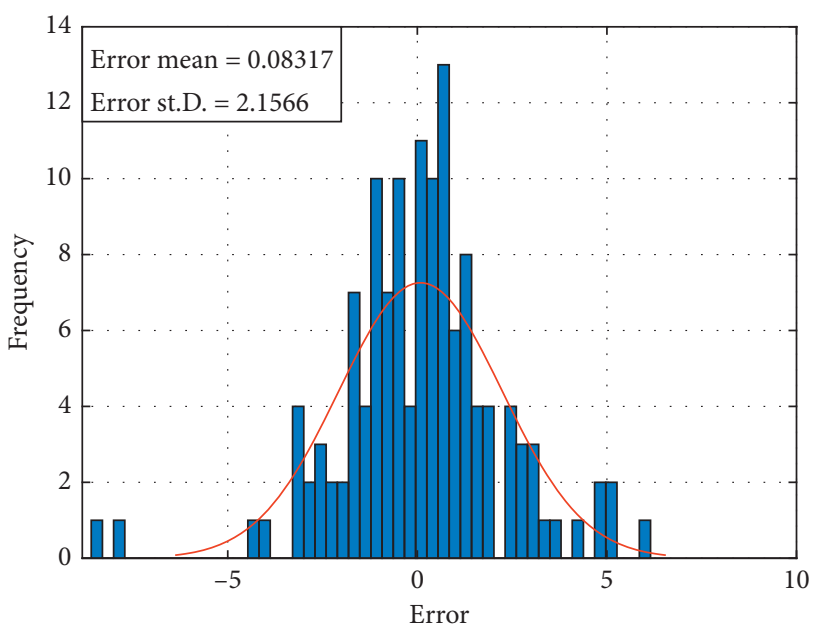

Figure 7: Histogram of error for the training dataset and a fit corresponds to the distribution of error.

respectively. Overall, FNN is a promising algorithm to predict the LACS of concrete.

4.2. FNN Typical Configuration Result. The results of a typical FNN configuration are presented in this section. Figure 5 shows the comparison between LACS results of the FNN model and the experimental data for the training dataset. The comparison shows that the predicted results were very close to the experimental results. Figure 6 shows that the FNN algorithm can successfully predict the experimental compressive strength for the testing dataset.

The histograms of error with respect to the training and testing parts are displayed in Figures 7 and 8, respectively. The mean errors were 0.08317 for the training part and 1.3724 for the testing part. The standard deviations of error values were computed as 2.1566 and 2.5211 for the training

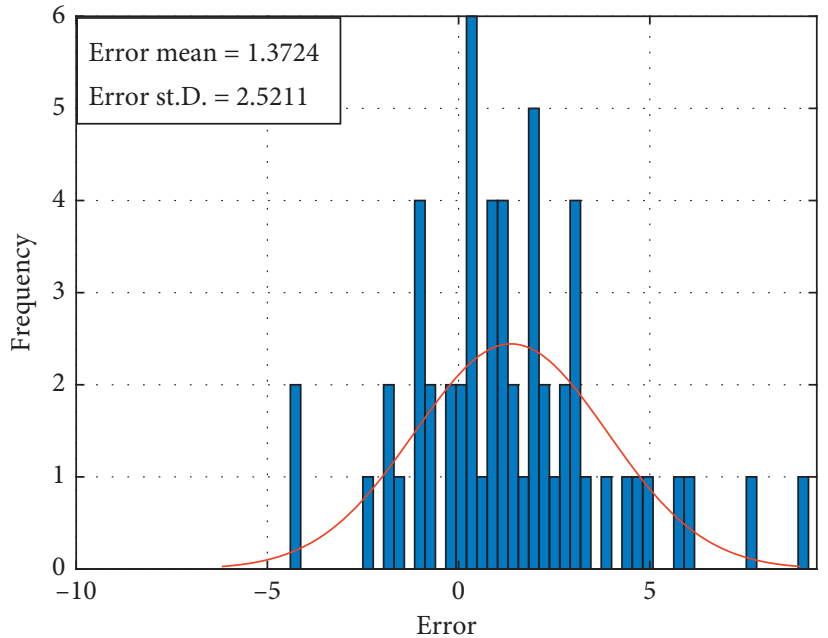

Figure 8: Histogram of error for the testing dataset and a fit corresponds to the distribution of error.

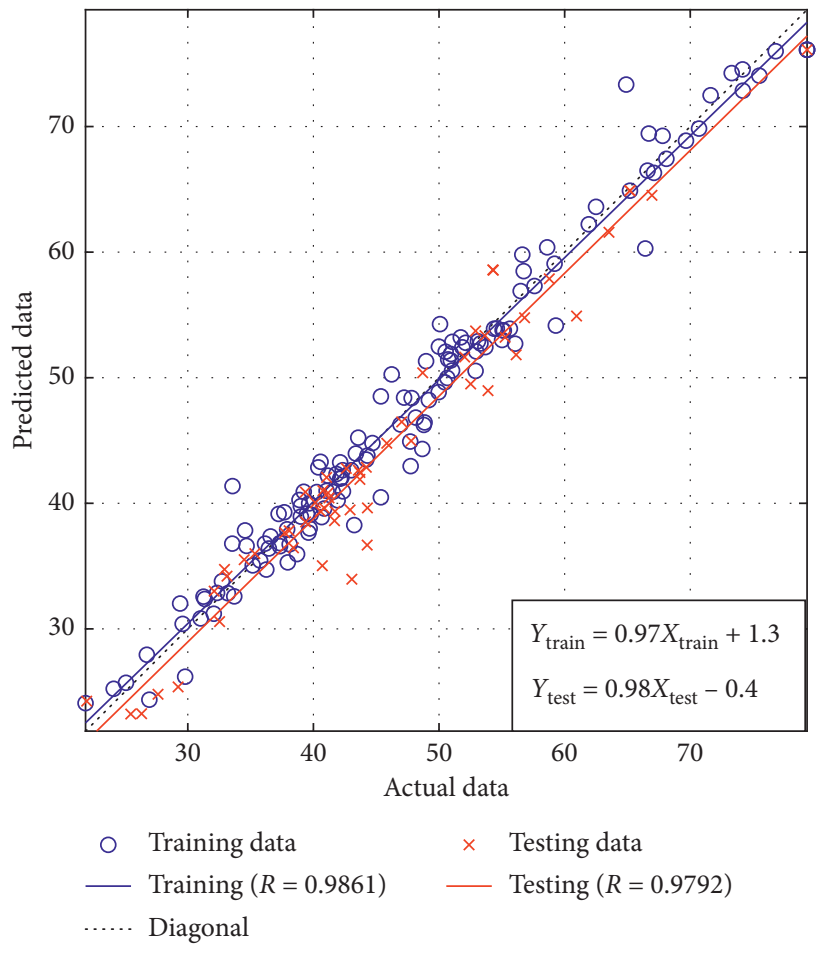

FIGURE 9: Regression graph for the training and testing datasets using the FNN model.

and testing datasets, respectively. It can be seen that the accuracy of the training part was slightly superior to the testing part, but both results showed excellent accuracy.

Finally, the relationships of the actual and predicted data were given in the form of regression plots in Figure 9. The values of $R$ were 0.9861 and 0.9792 for the training and testing datasets, respectively. Two equations related to such relationships were given in the graph, where " $Y=0.97 X+1.3$ " was the best fit line for the training set and " $Y=0.98 X-0.4$ " was the best fit linear regression for the testing set. 


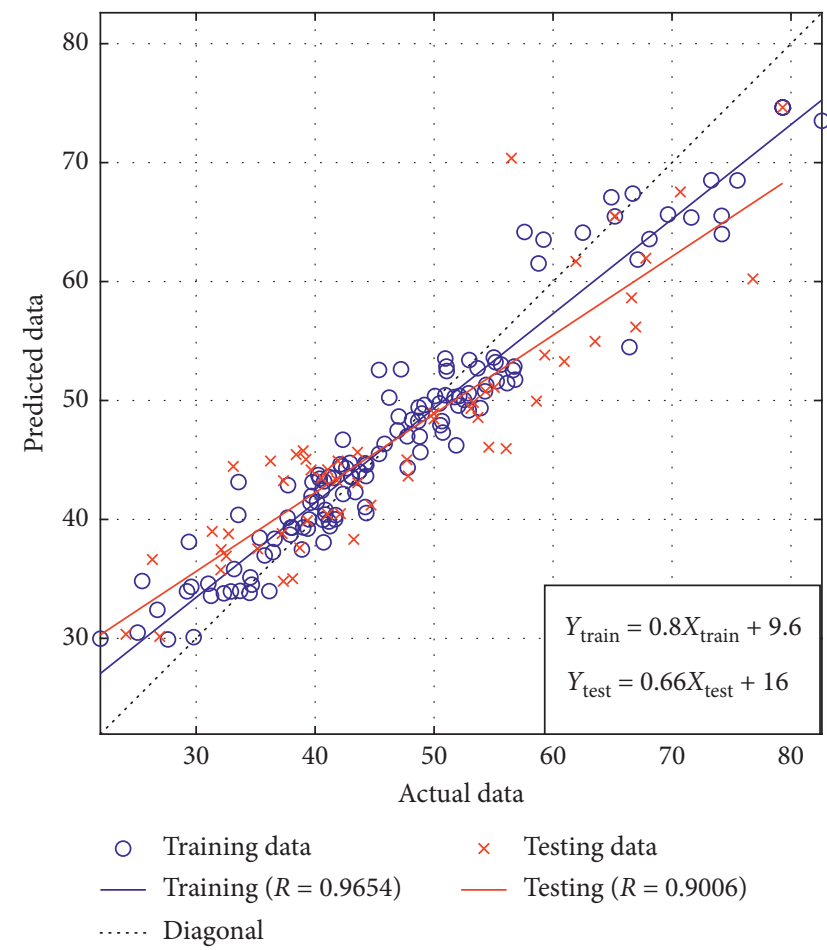

(a)

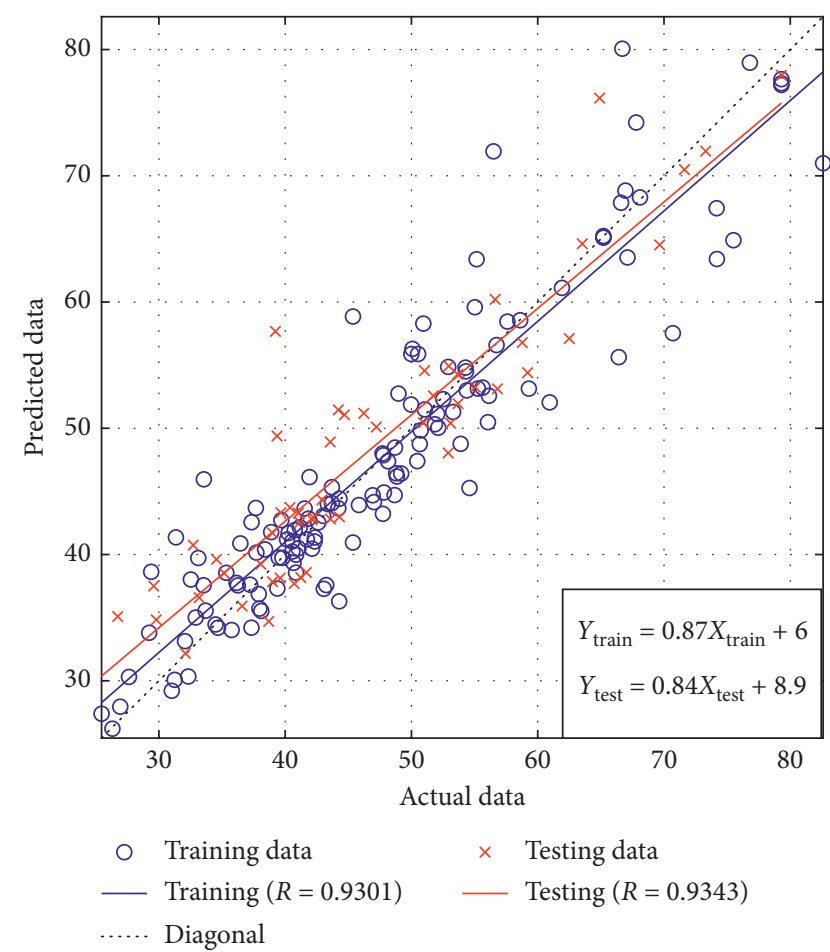

(b)

FIGURE 10: Regression graphs for the training and testing datasets for (a) RF algorithm and (b) GPR algorithm.

TABLE 4: Performance analysis of FNN, RF, and GPR algorithms.

\begin{tabular}{lccc}
\hline & FNN & RF & GPR \\
\hline Training dataset & & & \\
$R$ & 0.9861 & 0.9654 & 0.9301 \\
RMSE & 2.1501 & 3.6900 & 4.6411 \\
$\quad$ MAE & 1.5650 & 2.7728 & 3.1891 \\
\hline Testing dataset & & & \\
$R$ & 0.9792 & 0.9006 & 0.9343 \\
RMSE & 2.8510 & 6.2663 & 4.9469 \\
MAE & 2.1361 & 5.0560 & 3.7502 \\
\hline
\end{tabular}

The FNN algorithm showed an excellent performance in predicting the LACS of concrete. It is worth noticing that the values of $R$ could reach 0.9792 with the use of a simple FNN structure FNN (8-10-1), representing one hidden layer and ten neurons in such hidden layer.

For comparison purpose, the performance of FNN algorithm was shown superior to other benchmark machine learning algorithms, such as random forest (RF) and Gaussian process regression (GPR). The detail and development of RF and GPR algorithms are described in [37, 38], respectively. The regression graphs for the training and testing datasets of RF and GPR algorithms are shown in Figure 10. A summarized performance of FNN, RF, and GPR algorithms is shown in Table 4.

Overall, the FNN algorithm exhibited the best prediction performance with respect to RMSE, MAE, and $R$, compared with RF and GPR algorithms. Therefore, the FNN algorithm appears as a promising numerical tool to predict the laterage compressive strength of concrete.

\section{Conclusion}

In this study, the FNN algorithm was introduced to predict the later-age compressive strength of concrete containing cement, fly ash, blast furnace slag, water, and aggregates. A number of 190 experimental results were collected to construct the FNN model. A number of $70 \%$ of data was randomly chosen for the training phase of $\mathrm{FNN}$, and $30 \%$ of data was chosen for the validation phase of the constructed FNN model. To fully evaluate the performance of FNN, a number of 1000 simulations were performed using a random sampling technique, and the evaluation of FNN model was carried out by common statistical measurements.

The results showed that the FNN was a promising algorithm to predict the LACS of concrete, with the average values of $0.9350,4.3342$, and 3.2552 for $R$, RMSE, and MAE, respectively, for the testing part. The most accurate FNN model exhibited an excellent prediction performance with $R=0.9792$, $\mathrm{RMSE}=2.8510$, and $\mathrm{MAE}=2.1361$. These results were shown superior to other classical machine learning algorithms, namely, RF and GPR. The results could help in constructing a reliable soft computing tool to predict accurately and quickly the later-age compressive strength of concrete. Once the tool is built, the forecast process would take a millisecond to estimate the compressive strength of the given mix design. 


\section{Data Availability}

The processed data are available from the corresponding author upon request.

\section{Conflicts of Interest}

The authors declare that they have no conflicts of interest.

\section{References}

[1] V. Q. Tran, A. Soive, S. Bonnet, and A. Khelidj, "A numerical model including thermodynamic equilibrium, kinetic control and surface complexation in order to explain cation type effect on chloride binding capability of concrete," Construction and Building Materials, vol. 191, pp. 608-618, 2018.

[2] V. Q. Tran, A. Soive, and V. Baroghel-Bouny, "Modelisation of chloride reactive transport in concrete including thermodynamic equilibrium, kinetic control and surface complexation," Cement and Concrete Research, vol. 110, pp. 70-85, 2018.

[3] V. Q. Tran, H. L. Nguyen, V. D. Dao et al., "Temperature effects on chloride binding capacity of cementitious materials," Magazine of Concrete Research, pp. 1-14, 2019.

[4] A. Soive, V.-Q. Tran, and M. Gasc-Barbier, "The advantages of using a geochemical transport model including thermodynamic equilibrium, kinetic control and surface complexation to simulate the durability of concretes exposed to chlorides and sulphates," European Journal of Environmental and Civil Engineering, vol. 23, no. 5, pp. 552-563, 2019.

[5] A. Soive, V. Q. Tran, and V. Baroghel-Bouny, "Requirements and possible simplifications for multi-ionic transport models-case of concrete subjected to wetting-drying cycles in marine environment," Construction and Building Materials, vol. 164, pp. 799-808, 2018.

[6] A. Soive and V. Q. Tran, "External sulfate attack of cementitious materials: new insights gained through numerical modeling including dissolution/precipitation kinetics and surface complexation," Cement and Concrete Composites, vol. 83, pp. 263-272, 2017.

[7] V. Q. Tran, Contribution à la compréhension des mécanismes de dépassivation des armatures d'un béton exposé à l'eau de mer: théorie et modélisation thermochimique, Ph.D. thesis, École Centrale de Nantes, Nantes, France, 2016.

[8] M. Hasan and A. Kabir, "Prediction of compressive strength of concrete from early age test result," in Proceedings of the 4th Annual Paper Meet and 1st Civil Engineering Congress, Dhaka, Bangladesh, December 2011.

[9] S. V. Deodhar, Civil Engineering Materials, Khanna Publishers, Delhi, India, 6th edition, 2009.

[10] L. Zongjin, Advanced Concrete Technology, John Wiley \& Sons, Inc, Hoboken, NJ, USA, 1st edition, 2011.

[11] M. S. Shetty, Concrete Technology: Theory and Practice, MultiColor Illustration Edition and S. Chand, New Delhi, India, 2005.

[12] M. O. Reilly, D. Darwin, J. Sperry, and J. Browning, "Variation of concrete strength, permeability, and porosity due to specimen," The Kansas Department of Transportation, Topeka, KS, USA, 2017.

[13] J. Meader, D. Janssen, and M. Eberhard, Structural Design Parameters of Current WSDOT Mixtures, National Transportation Library, Washington, DC, USA, 2013.

[14] H.-B. Ly, B. Pham, Dao, L. Le, Le, and Le, "Improvement of ANFIS model for prediction of compressive strength of manufactured sand concrete," Applied Sciences, vol. 9, no. 18, p. 3841, 2019.

[15] L. M. Le, H.-B. Ly, B. T. Pham et al., "Hybrid artificial intelligence approaches for predicting buckling damage of steel columns under axial compression," Materials, vol. 12, no. 10, p. 1670, 2019.

[16] H.-B. Ly, L. M. Le, H. T. Duong et al., "Hybrid artificial intelligence approaches for predicting critical buckling load of structural members under compression considering the influence of initial geometric imperfections," Applied Sciences, vol. 9, no. 11, p. 2258, 2019.

[17] H. Q. Nguyen, H.-B. Ly, V. Q. Tran, T.-A. Nguyen, T.-T. Le, and B. T. Pham, "Optimization of artificial intelligence system by evolutionary algorithm for prediction of axial capacity of rectangular concrete filled steel tubes under compression," Materials, vol. 13, no. 5, p. 1205, 2020.

[18] D. Dao, H.-B. Ly, S. Trinh, T.-T. Le, and B. Pham, “Artificial intelligence approaches for prediction of compressive strength of geopolymer concrete," Materials, vol. 12, no. 6, p. 983, 2019.

[19] D. J. Montana and L. Davis, "Training feedforward neural networks using genetic algorithms," Proceedings of the 11th International Joint Conference on Artificial Intelligence -, vol. 1, no. 89, pp. 762-767, 1989.

[20] H.-G. Ni and J.-Z. Wang, "Prediction of compressive strength of concrete by neural networks," Cement and Concrete Research, vol. 30, no. 8, pp. 1245-1250, 2000.

[21] Q. H. Nguyen, H.-B. Ly, V. Q. Tran et al., "A novel hybrid model based on a feedforward neural network and one step secant algorithm for prediction of load-bearing capacity of rectangular concrete-filled steel tube columns," Molecules, vol. 25, no. 15, p. 3486, 2020.

[22] H. Adeli, "Neural networks in civil engineering: 1989-2000," Computer-Aided Civil and Infrastructure Engineering, vol. 16, no. 2, pp. 126-142, 2001.

[23] A. Mukherjee and S. Nag Biswas, "Artificial neural networks in prediction of mechanical behavior of concrete at high temperature," Nuclear Engineering and Design, vol. 178, no. 1, pp. 1-11, 1997.

[24] P. Muthupriya, K. Subramanian, and B. G. Vishnuram, "Prediction of compressive strength and durability of high performance concrete by artificial neural networks," International Journal of Optimization in Civil Engineering, vol. 1, pp. 189-209, 2011.

[25] A. Khalaf, Z. Naser, and F. Idan, "Predicting the ultimate strength of circular concrete filled steel tubular columns by using artificial neural networks," pp. 9-16, 2018.

[26] A. M. Al-Khaleefi, M. J. Terro, A. P. Alex, and Y. Wang, "Prediction of fire resistance of concrete filled tubular steel columns using neural networks," Fire Safety Journal, vol. 37, no. 4, pp. 339-352, 2002.

[27] Y. N. Chan, P. Jin, M. Anson, and J. S. Wang, "Fire resistance of concrete: prediction using artificial neural networks," Magazine of Concrete Research, vol. 50, no. 4, pp. 353-358, 1998.

[28] M. Sazli, "A brief review of feed-forward neural networks," Communications, Faculty of Science, University of Ankara, vol. 50, pp. 11-17, , Ankara, Turkey, 2006.

[29] H.-B. Ly, T.-T. Le, L. M. Le et al., "Development of hybrid machine learning models for predicting the critical buckling load of I-shaped cellular beams," Applied Sciences, vol. 9, no. 24, p. 5458, 2019.

[30] H.-B. Ly, L. M. Le, L. V. Phi et al., "Development of an AI model to measure traffic air pollution from multisensor and weather data," Sensors, vol. 19, no. 22, p. 4941, 2019. 
[31] I.-C. Yeh, "Modeling concrete strength with augment-neuron networks," Journal of Materials in Civil Engineering, vol. 10, no. 4, pp. 263-268, 1998.

[32] I.-C. Yeh, "Design of high-performance concrete mixture using neural networks and nonlinear programming," Journal of Computing in Civil Engineering, vol. 13, no. 1, pp. 36-42, 1999.

[33] I.-C. Yeh, "A mix proportioning methodology for fly ash and slag concrete using artificial neural networks," Chung Hua Journal of Science and Engineering, vol. 1, pp. 77-84, 2003.

[34] I.-C. Yeh, "Analysis of strength of concrete using design of experiments and neural networks," Journal of Materials in Civil Engineering, vol. 18, no. 4, pp. 597-604, 2006.

[35] I.-C. Yeh, "Modeling of strength of high-performance concrete using artificial neural networks," Cement and Concrete Research, vol. 28, no. 12, pp. 1797-1808, 1998.

[36] H.-B. Ly, E. Monteiro, T.-T. Le et al., "Prediction and sensitivity analysis of bubble dissolution time in $3 \mathrm{D}$ selective laser sintering using ensemble decision trees," Materials, vol. 12, no. 9, p. 1544, 2019.

[37] T. A. Pham, H.-B. Ly, V. Q. Tran, L. V. Giap, H.-L. T. Vu, and H.-A. T. Duong, "Prediction of pile axial bearing capacity using artificial neural network and random forest," Applied Sciences, vol. 10, no. 5, p. 1871, 2020.

[38] D. V. Dao, H. Adeli, H.-B. Ly et al., "A sensitivity and robustness analysis of GPR and ANN for high-performance concrete compressive strength prediction using a monte carlo simulation," Sustainability, vol. 12, no. 3, p. 830, 2020. 